\title{
ANALYSIS OF FLOW DETACHMENT IN THE INTAKE MANIFOLD OF A FORMULA SAE VEHICLE
}

\author{
Ivan Sanches Provase, Prof. Dr. Marcos M. Pimenta, Prof Dr. Antônio L. C. Mariani \\ ESCOLA POLITÉCNICA DA UNIVERSIDADE DE SÃO PAULO
}

provase.ivan@gmail.com , camposmariani@gmail.com, mmpimenta@uol.com.br

\section{Resumo}

Durante testes, o motor do veículo de Fórmula SAE da Escola Politécnica da Universidade de São Paulo apresentou oscilações em sua rotação, quando operando em alta rotação e alta vazão mássica. Decidiu-se então entender e por fim, solucionar o problema, começando pela análise detalhada do sistema de admissão do mesmo. O estudo do escoamento no duto de admissão de um veículo de competição se torna extremamente relevante ao passo que, dentro das limitações construtivas e limitações impostas pelas regras da competição de Fórmula SAE, tenta-se melhorar ao máximo o desempenho do mesmo. Somando-se as prerrogativas de alto desempenho do motor, com o espaço disponível para fixação do mesmo ao veículo, o runner, peça crítica no quesito desempenho, acaba por apresentar uma acentuada curvatura. Esta que se torna relevante principalmente quando o motor opera em altas rotações e altas vazões mássicas, já que nestas condições, pode-se ter o descolamento do fluido da parede interna. Assim, o comportamento fluídico do ar no sistema de admissão do veículo de fórmula SAE da Escola Politécnica de São Paulo foi estudado. Um modelo em CFD foi feito e simulado em regime permanente, visando analisar principalmente o campo de velocidade. Este, por fim, indicou a presença de recirculação do ar na região interna da curvatura do runner; provocando assim uma diminuição da seção transversal útil para escoamento do ar; que por sua vez, causou uma diminuição na oferta de torque do motor, justificando assim as oscilações na rotação que o motor apresentava.

\section{Introdução}

O presente trabalho baseia-se no estudo do escoamento de fluido que ocorre na admissão de um motor de combustão interna de um veículo utilizado na Fórmula SAE.

O motor e os seus periféricos de um veículo estão sujeitos a dimensões máximas impostas pela estrutura do veículo. A admissão do ar pelo motor ocorre através de um duto de admissão usualmente identificado como runner. Este acessório é definido como o primeiro duto entre o flange do motor e um plenum. O plenum fica caracterizado pela região entre o runner e o corpo de borboleta, responsável por alimentar o runner. O ideal seria adotar-se este runner como um tubo reto, mas há várias limitações que inviabilizam esta alternativa. $\mathrm{O}$ plenum, devido ao seu significativo volume, tende-se a moldar com a estrutura do veículo, tendo que respeitar limites determinados pela região do cofre do motor. Neste contexto o runner também acaba ganhando uma curvatura que, em muitos casos, é acentuada.

O estudo de caso que foi a motivação deste projeto e que será adotado nas análises é o veículo de Fórmula SAE projetado pela equipe Poli Racing da Escola Politécnica da USP. Este veículo de competição tem exigentes 
restrições e prerrogativas como: alta performance, motor monocilíndrico operando em rotações próximas a treze mil rotações por minuto. Como consequência das condições de operação o escoamento na região do runner, possui gradientes de pressão e velocidades significativamente altos, atingindo valores para o número Mach maiores que 0.3 , na admissão.

Durante observação em condições de operação, o veículo de Fórmula SAE apresentou instabilidades em parâmetros associados ao fluxo de ar, relacionadas às variações na rotação do motor. Um possível causador dessa instabilidade pode ser o descolamento do fluido da parede interna do runner, que possui curvatura acentuada em relação ao diâmetro do mesmo, e seu estudo estará no foco deste projeto.

\section{Projeto do runner}

As dimensões do runner de um motor de competição são definidas por dois principais aspectos. O primeiro diz respeito às dimensões máximas e ao formato do cofre do motor ou região em que o mesmo se encontra. $\mathrm{O}$ outro diz respeito à região de rotação em que se deseja que a ressonância do ar de admissão aconteça com maior intensidade, aumentando assim a eficiência volumétrica do motor naquela região.

A competição de Fórmula SAE em 2011, determinava que nenhuma parte do veículo em questão deveria estar fora do envelope secundário, definido pela altura mínima prevista por regra, o conjunto de rodas e o tubo do main hoop, conforme visto na figura.

SIDE VIEW

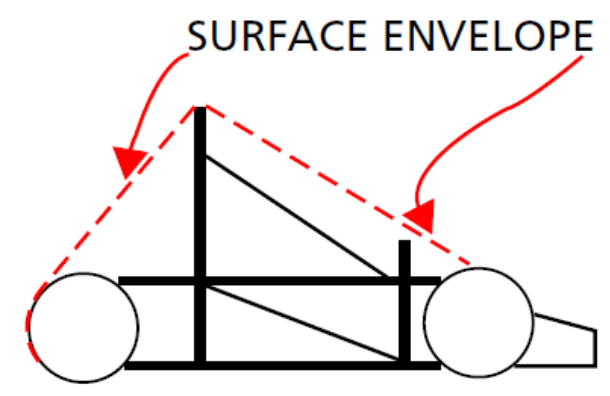

REAR VIEW

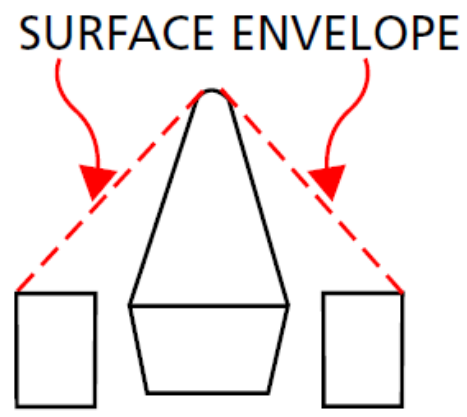

Figura 1. Envelope secundário definido pela regra de 2011 da competição de Fórmula SAE [1]

Desta maneira, o motor, que se encontra na região traseira do veículo, assim como seus periféricos tem dimensões limitadas para ocupar.

O segundo aspecto levantado diz respeito ao fenômeno físico de ressonância que ocorre no duto de admissão de qualquer motor. $\mathrm{O}$ abrir e fechar as válvulas de admissão criam um fluxo pulsante de ar no duto de admissão. Além disso, os gradientes de pressão ali gerados formam ondas que se propagam no duto, em ambas as direções. Conforme descrito em Heywood [3], pode-se tirar proveito de tais fenômenos de ressonância umas vez que estas ondas podem ser divididas em dois tipos de ondas: ondas de rarefação e ondas de alta pressão. $\mathrm{O}$ abrir da válvula de admissão cria uma onda de rarefação que se desloca no sentido contrário do escoamento do fluido, indo da jusante para a montante, ou seja, da válvula de admissão para o plenum. Ao encontrar o plenum, a mesma é refletida de volta na direção contrária, da montante para jusante, como uma onda de sobre pressão. Nota-se que para isso, a geometria da conexão entre o runner e o plenum deve ser corretamente construída, ou seja, uma abertura única sem posterior contração, conforme visto na parte inferior da figura 2. 


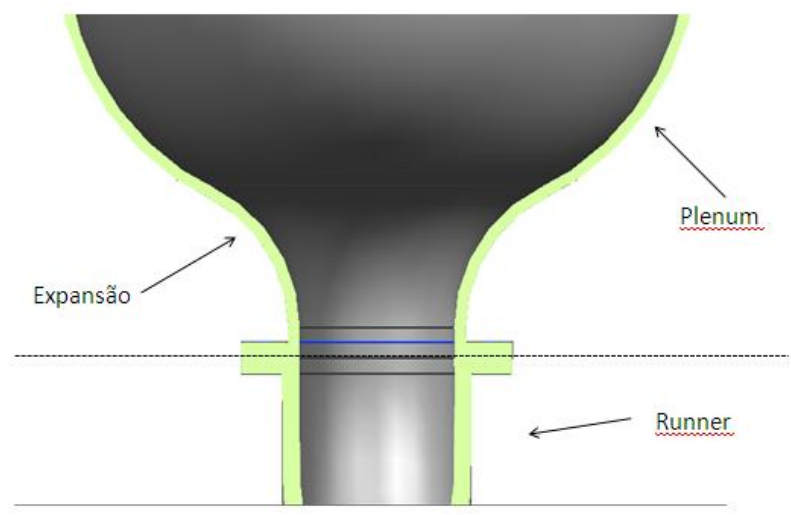

Figura 2. Corte longitudinal da admissão de ar enfatizando a conexão entre o runner e o plenum [2]

Assim, o conjunto: cilindro + runner + plenum deve ser corretamente projetado para que esta onda de sobre pressão encontre a válvula de admissão aberta, fazendo com que mais ar seja admitido, simulando uma indução forçada, aumentando assim a eficiência volumétrica do motor.

J. B. Heywood descreveu tal fenômeno e equacionou que a rotação do motor (monocilíndrico) em que tal ressonância (sincronização da chegada das ondas de alta pressão com a abertura das válvulas) ocorre é dada por:

$$
N=\frac{955}{K} a\left(\frac{A}{l \cdot V_{e f f}}\right)^{1 / 2}
$$

Com

$$
V_{e f f}=V_{d} \frac{\left(r_{c}+1\right)}{2\left(r_{c}-1\right)}
$$

Em que:

$V_{d}-$ volume do cilindro

$r_{c}$ - razão de compressão

a - velocidade do som

A - área da seção transversal do runner

$l$ - comprimento do runner

Assim, ficam explicitas as duas principais relações. A primeira, uma relação termodinâmica entre a frequência de ressonância e, por conseguinte, a rotação de ressonância, e o fluido operante. Tal relação é mostrada na dependência da rotação de ressonância e a velocidade do som, esta que depende da temperatura do fluido e também do coeficiente de expansão isotrópico (igual a 1.4 para moléculas diatômicas, como no caso do ar, constituído por $\mathrm{O}_{2}$ e $\mathrm{N}_{2}$ basicamente), conforme mostrado na equação 3 . 


$$
a=\sqrt{k \cdot R \cdot T}
$$

$\mathrm{k}$ - coeficiente de expansão isotrópico

$\mathrm{T}$ - temperatura

$\mathrm{R}$ - constante universal dos gases

A segunda, uma relação geométrica entre o volume da câmara de combustão, a razão de compressão, a área do runner e o comprimento do mesmo. Uma vez que o motor, sua câmara de combustão e razão de compressão sejam projetados seguindo diretrizes mais relevantes que a frequência de ressonância do mesmo (homogeneidade da mistura, otimização do fluxo médio em todas as regiões de operação, evitar a pré-ignição e knocking, entre outros), cabe ao projeto do runner garantir que a ressonância ocorra na região esperada para o uso do motor (que a priori varia de acordo com a competição / finalidade do motor). A área da seção transversal muitas vezes já é definida, uma vez que, escolhido o motor, esta deve ser igual à área do flange do motor, para evitar perda de carga localizada (causada pela abrupta contração / expansão na seção transversal de escoamento do fluido).

Assim, a principal variável para determinar a frequência de ressonância que o projetista do runner possui é o comprimento do mesmo.

Tendo essas premissas, e com a rotação de interesse de 8500 RPM definida, o runner final projetado terminou por possuir $40 \mathrm{~mm}$ de diâmetro e um raio de curvatura de $85 \mathrm{~mm}$ que se estendem por $113^{\circ}$, conferindo a este um comprimento de aproximadamente $168 \mathrm{~mm}$ (comprimento da linha meridional entre os dois flanges).

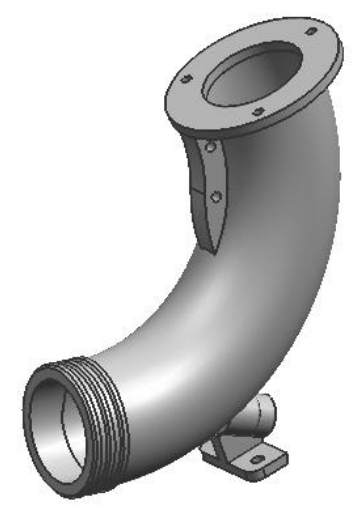

Figura 3. Runner projetado pela equipe Poli Racing de Fórmula SAE [2]

A figura 4 mostra em uma vista lateral, os detalhes do posicionamento do motor e do sistema de admissão do veículo de Fórmula SAE da Escola Politécnica da Universidade de São Paulo, mostrando também parte da estrutura e roda traseira esquerda, enfatizando como todo o conjunto, motor e entrada de ar, se encontram dentro do envelope secundário. 


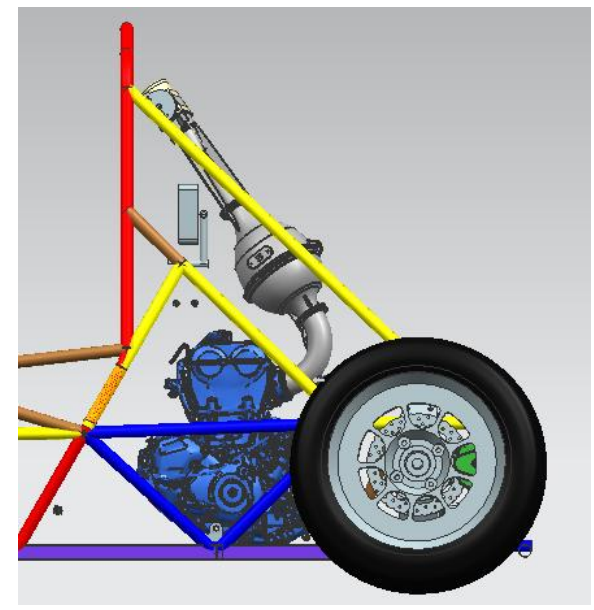

Figura 4. Imagem da montagem do motor e admissão de ar, mostrando que o conjunto se encontra dentro do envelope secundário do veículo [2]

\section{Descrição do fenômeno físico do descolamento do ar}

A contraposição do projeto do runner orientado segundo as duas premissas acima descritas, dimensões máximas da região do motor e frequência de ressonância para aumentar a eficiência volumétrica, está na consequência fluídica que estas impõem ao escoamento. Seja qual for o fluido operante, o mesmo possui uma determinada viscosidade e massa específica, assim, mudanças na direção do escoamento, como no caso do runner, a sua curvatura, geram consequências.

Além dos escoamentos secundários criados numa mudança de direção do escoamento devido à conservação da quantidade de movimento, que não serão tratados aqui, a simples inércia do fluido e viscosidade podem gerar consequências graves para o escoamento. Se a mudança de direção for abrupta o suficiente e o número de Reynolds do escoamento alto suficiente, indicando que as forças de inércia no escoamento se sobrepõem às forças viscosas, o mesmo pode descolar da parede interna do duto, na região interna da curvatura do duto.

Tal descolamento faz com que a seção transversal útil para o escoamento seja reduzida, uma vez que a porção interna da curva do duto possui fluido recirculante ou em alguns casos, o fluido se encontra praticamente estagnado nessa região. Tal fenômeno fica claro na figura abaixo, retirada de Munson [4], que mostra a relação do fator de perda de carga, Kl, em função da razão entre o raio de curvatura do conduto e o seu diâmetro.

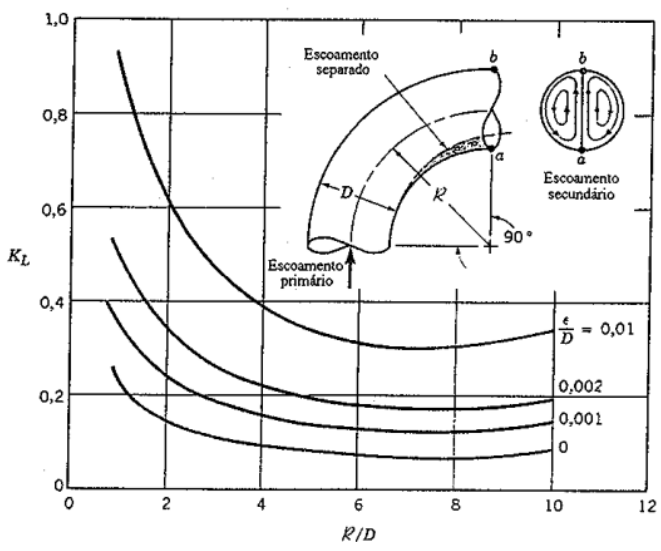

Figura 5. Diagrama da perda de carga em função da razão do raio de curvatura e do diâmetro do conduto 
Ao passo que a redução do raio de curvatura gera uma perda singular de carga no escoamento devido ao descolamento / recirculação do fluido, o aumento excessivo deste também não é desejável pois assim, aumentam-se as perdas distribuídas de carga.

A redução da seção transversal útil para o escoamento do fluido é extremamente danosa para o motor, uma vez que isso significa que menos ar poderá ser admitido e, portanto, a potência do mesmo será limitada.

\section{Simulação numérica}

A fim de analisar um escoamento por completo, 3 grupos de equações devem ser consideradas: a equação da conservação da massa, a equação da conservação da quantidade de movimento (também conhecida como equações de Navier-Stokes) e a equação da conservação da energia. Tais equações descrevem o comportamento do fluido e da condução térmica no mesmo, nas três dimensões ao longo do tempo, para qualquer posição do fluido; conforme pode ser visto abaixo, respectivamente, quando expressadas de maneira mais genérica (nenhuma hipótese simplificadora aplicada):

$$
\begin{gathered}
\frac{\partial \rho}{\partial \mathrm{t}}+\nabla(\rho \mathrm{u})=0 \\
\frac{\mathrm{D}(\rho \mathrm{u})}{\mathrm{Dt}}+\mathrm{u} \cdot \nabla(\rho \mathrm{u})+\nabla \mathrm{p}+\nabla \cdot \mathrm{T}-\mathrm{f}=0 \\
\partial \mathrm{Q}-\mathrm{dU}-\partial \mathrm{W}=0
\end{gathered}
$$

Em que:

$\mathrm{u}$ - velocidade do ponto $[\mathrm{u}=\mathrm{u}(\mathrm{x}, \mathrm{y}, \mathrm{z}, \mathrm{t})]$

$\mathrm{p}-$ pressão no ponto $[\mathrm{p}=\mathrm{p}(\mathrm{x}, \mathrm{y}, \mathrm{z}, \mathrm{t})]$

$\rho$-densidade do fluido no ponto $[\rho=\rho(\mathrm{x}, \mathrm{y}, \mathrm{z}, \mathrm{t})]$

$\mathrm{T}$ - tensor total das tensões

f - forças de campo (gravidade, forças eletromagnéticas, etc...)

Q - quantidade de calor transferido para o sistema

$\mathrm{U}$ - energia interna do sistema

$\mathrm{W}$ - trabalho realizado pelo sistema

Fica claro que o tratamento genérico destas equações traz consigo uma enorme quantidade de grandezas a serem consideradas. Desta maneira, é necessário que algumas considerações, sejam elas físicas, ou mesmo matemáticas, sejam feitas para possibilitar a resolução das mesmas.

Simplificações matemáticas, como a linearização de alguns termos, devem então ser feitas para permitir a solução das equações, impondo porém um problema intrínseco de tal ação: uma vez linearizadas, as equações deixam de descrever com precisão os pontos de inflexão, assim como pontos de ruptura (no caso do estudo dos escoamentos, descolamentos de parede por exemplo), tornando o pós tratamento de dados uma tarefa 
extremamente importante e complexa, visando com esta, validar as condições e resultados gerados pelas equações.

Deve-se ressaltar que, as limitações aqui apresentadas, não se restringem apenas à perda de informação sobre o escoamento, mas também, à capacidade computacional demandada.

Tendo essa visão simplificada da problemática, pode-se entender o porquê do uso de modelos de turbulência para descrever o escoamento: trata-se de modelos que pressupõem simplificações físicas (que englobam as propriedades do fluido, assim como condições de contorno) e matemáticas (linearizações das equações em torno de um ponto) para abordar determinado escoamento.

O RANS, Reynolds-averaged Navier-Stokes: apesar de ser uma das abordagens mais antigas no que diz respeito à modelagem de turbulência, é hoje amplamente utilizada devido ao seu sucesso: um bom comprometimento entre precisão e demanda computacional. Consiste em aplicar a chamada decomposição de Reynolds, reescrevendo as variáveis do fluido como uma componente média mais a sua flutuação. Por exemplo, o vetor velocidade seria dividido em duas parcelas: a velocidade média e sua oscilação. Desta forma:

$$
\mathrm{u}_{\mathrm{i}}=\mathrm{U}_{\mathrm{i}}+\mathrm{u}_{\mathrm{i}}^{\prime}
$$

Ou de maneira mais clara:

$$
\mathrm{u}(\mathrm{x}, \mathrm{t})=\mathrm{U}(\mathrm{x})+\mathrm{u}^{\prime}(\mathrm{x}, \mathrm{t})
$$

A utilização desta decomposição permite que o escoamento turbulento possa ser em parte tratado como um escoamento não turbulento (mas sim como um escoamento laminar no que diz respeito ao escoamento médio), facilitando sua resolução. O problema gerado por tal decomposição reside na chamada tensão de Reynolds. Apesar de não ser propriamente uma tensão, apenas possuir a unidade de tensão, tal tensor de segunda ordem é altamente não linear, e diferentes abordagens para o tratamento do mesmo geram resultados diferentes. Tal tensor é definido na equação 9.

$$
\tau_{\mathrm{ij}}=\rho \overline{\mathrm{u}_{1}^{\prime} \mathrm{u}_{\mathrm{j}}^{\prime}}
$$

Podemos com este novo enfoque, reescrever as equações que regem o escoamento de um fluido de outra maneira (ainda que genérica, mas sem considerar as forças de campo e admitindo um referencial inercial e introduzindo a decomposição de Reynolds):

$$
\begin{gathered}
\frac{\partial \rho}{\partial t}+\frac{\partial\left(\rho u_{i}\right)}{\partial x_{j}}=0 \\
\frac{\partial\left(\rho u_{i}\right)}{\partial t}+\frac{\partial\left(\rho u_{i} u_{j}\right)}{\partial x_{j}}-\frac{\partial \tau_{i j}}{\partial x_{j}}+\frac{\partial P}{\partial x_{i}}=0 \\
\frac{\partial\left(\rho e_{0}\right)}{\partial t}+\frac{\partial\left(\rho u_{i} e_{0}+\rho u_{j}+q_{j}-u_{i} \tau_{i j}\right)}{\partial x_{j}}-\frac{\partial P}{\partial t}=0
\end{gathered}
$$

Em que:

$\tau_{\mathrm{ij}}$ - Tensor das tensões de Reynolds

E sem perda de generalidade, podemos descrever a transferência de calor como: 


$$
\mathrm{q}_{\mathrm{j}}=-\lambda \frac{\partial \mathrm{T}}{\partial \mathrm{x}_{\mathrm{j}}}
$$

E a energia total como:

$$
\mathrm{e}_{0}=\mathrm{e}+\frac{\mathrm{u}_{\mathrm{k}} \mathrm{u}_{\mathrm{k}}}{2}
$$

A utilização da decomposição de Reynolds e do tensor de Reynolds impõem um problema de fechamento (BBGKY) [5]: o número de equações é menor do que o número de incógnitas, tornando impossível a resolução das mesmas.

Assim, uma segunda hipótese pode ser adotada, a hipótese de Boussinesq. Nesta, faz-se uso de equações algébricas para a tensão de Reynolds, incluindo a determinação da viscosidade turbulenta, resolvendo as equações de transporte para grandezas relevantes. Aqui residem grandes partes dos modelos: modelos de zero (Cebeci-Smith ou Baldwin-Lomax, por exemplo), uma (modelo de Prandtl, Baldwin-Barth ou Spalart-Allmaras, por exemplo) ou duas (k-w ou k-e, por exemplo) equações.

A base para os modelos reside na hipótese de Boussinesq para a viscosidade turbulenta, que postula o tensor das tensões de Reynolds, $\tau_{\mathrm{ij}}$, como proporcional ao tensor de deformação média, $\mathrm{S}_{\mathrm{ij}}$, e pode ser descrito como:

$$
\tau_{\mathrm{ij}}=2 \mu_{\mathrm{t}} \mathrm{S}_{\mathrm{ij}}-\frac{2}{3} \rho \frac{\overline{\mathrm{u}_{1}^{\prime} \mathrm{u}_{1}^{\prime}}}{2} \delta_{\mathrm{ij}}
$$

Ou reescrita como:

$$
-\rho \overline{u_{1}^{\prime} u_{j}^{\prime}}=\mu_{t}\left(\frac{\partial u_{j}}{\partial x_{i}}+\frac{\partial u_{i}}{\partial x_{j}}\right)-\frac{2}{3} \rho \frac{\overline{u_{1}^{\prime} u_{1}^{\prime}}}{2} \delta_{i j}
$$

Tal hipótese traz grande simplificação uma vez que permite pensar que o efeito da turbulência no escoamento médio, é semelhante ao efeito da viscosidade molecular no escoamento laminar, permitindo assim introduzir grandezas turbulentas escalares, como a energia turbulenta e a dissipação turbulenta, além de tornar possível a correlação destas com variáveis ainda mais intuitivas, como a intensidade turbulenta:

$$
\mathrm{I} \equiv \frac{\mathrm{u}^{\prime}}{\mathrm{U}}
$$

Com $\mathrm{u}^{\prime}$ representando a raiz quadrada média das oscilações turbulentas de velocidade e U representando a velocidade média do escoamento.

$$
u^{\prime} \equiv \sqrt{\frac{1}{3}\left(\mathrm{u}_{\mathrm{x}}{ }^{\prime 2}+\mathrm{u}_{\mathrm{y}}{ }^{\prime 2}+\mathrm{u}_{\mathrm{z}}{ }^{\prime 2}\right)}=\sqrt{\frac{2}{3} \mathrm{k}}
$$

E

$$
\mathrm{U} \equiv \sqrt{\mathrm{U}_{\mathrm{x}}{ }^{2}+\mathrm{U}_{\mathrm{y}}{ }^{2}+\mathrm{U}_{\mathrm{z}}{ }^{2}}
$$




\section{Modelo de turbulência}

Na simulação deste trabalho, o modelo utilizado foi o modelo de duas equações SST (shear stress transport) kw. Este modelo é baseado no modelo $\mathrm{k}-\mathrm{w}$, porém, conforme o nome dele induz, tal modelo modifica a viscosidade turbulenta de maneira que ela leve em conta o transporte da turbulência principal, dando a este modelo uma vantagem em relação ao modelo padrão k-€ ou mesmo ao k-w padrão. Além disto, outras modificações como a utilização da difusão cruzada na equação de w, e fundindo uma função que garanta que o modelo de equações seja confiável na região próxima à parede, assim como no campo distante.

As equações de transporte para o modelo SST k-w podem ser vistas abaixo.

$$
\begin{aligned}
& \frac{\partial(\rho \mathrm{k})}{\partial \mathrm{t}}+\frac{\partial\left(\rho \mathrm{k} \mathrm{u}_{\mathrm{i}}\right)}{\partial \mathrm{x}_{\mathrm{i}}}=\frac{\partial\left[\Gamma_{\mathrm{k}} \frac{\partial \mathrm{k}}{\partial \mathrm{x}_{\mathrm{j}}}\right]}{\partial \mathrm{x}_{\mathrm{j}}}+\mathrm{G}_{\mathrm{k}}-\mathrm{Y}_{\mathrm{k}}+\mathrm{S}_{\mathrm{k}} \\
& \frac{\partial(\rho \mathrm{w})}{\partial \mathrm{t}}+\frac{\partial\left(\rho \mathrm{w} \mathrm{u}_{\mathrm{i}}\right)}{\partial \mathrm{x}_{\mathrm{i}}}=\frac{\partial\left[\Gamma_{\mathrm{w}} \frac{\partial \mathrm{w}}{\partial \mathrm{x}_{\mathrm{j}}}\right]}{\partial \mathrm{x}_{\mathrm{j}}}+\mathrm{G}_{\mathrm{w}}-\mathrm{Y}_{\mathrm{w}}+\mathrm{D}_{\mathrm{w}}+\mathrm{S}_{\mathrm{w}}
\end{aligned}
$$

Os termos da difusividade de k e w são os mesmo definidos no modelo k-w padrão acima, porém a viscosidade turbulenta passa a ser descrita como:

$$
\mu_{\mathrm{t}}=\frac{\rho \mathrm{k}}{\mathrm{w}} \frac{1}{\max \left[\frac{1}{\alpha^{*}}, \frac{\Omega \mathrm{F}_{2}}{\mathrm{a}_{1} \mathrm{w}}\right]}
$$

Onde:

$$
\Omega \equiv \sqrt{2 \Omega_{\mathrm{ij}} \Omega_{\mathrm{ij}}}
$$

Nesta variação do modelo os números de Prandtl apresentam a função de mistura:

$$
\begin{aligned}
& \sigma_{\mathrm{k}}=\frac{1}{\frac{\mathrm{F}_{1}}{\sigma_{\mathrm{k}, 1}}+\frac{\left(1-\mathrm{F}_{1}\right)}{\sigma_{\mathrm{k}, 1}}} \\
& \sigma_{\mathrm{w}}=\frac{1}{\frac{\mathrm{F}_{1}}{\sigma_{\mathrm{w}, 1}}+\frac{\left(1-\mathrm{F}_{1}\right)}{\sigma_{\mathrm{W}, 1}}}
\end{aligned}
$$

As funções de mistura $F_{1}$ e $F_{2}$, são dadas por:

$$
\mathrm{F}_{1}=\tanh \left(\phi_{1}^{4}\right)
$$

Onde:

$$
\phi=\min \left[\max \left(\frac{\sqrt{\mathrm{k}}}{0.09 \mathrm{wy}}, \frac{500 \mu}{\rho \mathrm{y}^{2} \mathrm{w}}\right), \frac{4 \rho \mathrm{k}}{\sigma_{\mathrm{w}, 2} \mathrm{D}_{\mathrm{w}}^{+} \mathrm{y}^{2}}\right]
$$

Em que:

$$
\mathrm{D}_{\mathrm{w}}^{+}=\max \left[2 \rho \frac{1}{\sigma_{\mathrm{w}, 2}} \frac{1}{\mathrm{w}} \frac{\partial \mathrm{k}}{\partial \mathrm{x}_{\mathrm{j}}} \frac{\partial \mathrm{w}}{\partial \mathrm{x}_{\mathrm{j}}}, 10^{-20}\right]
$$

Assim como:

Page 9 of 17 


$$
\mathrm{F}_{1}=\tanh \left(\phi_{2}^{2}\right)
$$

Com:

$$
\phi_{2}=\max \left[2 \frac{\sqrt{\mathrm{k}}}{0.09 \mathrm{wy}}, \frac{500 \mu}{\rho \mathrm{y}^{2} \mathrm{w}}\right]
$$

Em que nas equações a cima, y, refere-se à distância à superfície próxima e $\mathrm{D}_{\mathrm{w}}^{+}$é a porção positiva do modelo de difusão cruzada, $\mathrm{D}_{\mathrm{w}}^{+}$, uma consequência da utilização do modelo k-€ e da sua transformação em equações baseadas em w.

A difusividade cruzada é calculada como:

$$
\mathrm{D}_{\mathrm{w}}^{+}=2\left(1-\mathrm{F}_{1}\right) \rho \sigma_{\mathrm{w}, 2} \frac{1}{\mathrm{w}} \frac{\partial \mathrm{k}}{\partial \mathrm{x}_{\mathrm{j}}} \frac{\partial \mathrm{w}}{\partial \mathrm{x}_{\mathrm{j}}}
$$

\section{Condições de contorno}

Algumas hipóteses simplificadoras tiveram de ser feitas para garantir uma simulação mais precisa e um pós tratamento de dados mais confiável.

O fluido operante simulado foi o ar, a $25^{\circ} \mathrm{C}$ e 1 atm, uma vez que a simulação diz respeito à condição de vazão máxima, em que o corpo de borboleta está totalmente aberto e a pressão do plenum se iguala a pressão atmosférica. Quando em operação (pista de corrida) devido ao fato do motor não ser coberto por carenagem e as tomadas de ar laterais do veículo promoverem grande fluxo de ar na região traseiro do veículo, pode-se admitir que o ar no sistema de admissão está a uma temperatura próxima ao ar ambiente (mesmo que não seja exatamente a mesma temperatura, para o estudo aqui realizado, uma pequena diferença não é relevante)

A simulação foi feita em regime estacionário. Apesar de o escoamento real ser extremamente pulsante (a 8500 RPM, rotação de escolha da frequência de ressonância, o processo de admissão leva cerca de 8 ms) isso tornaria a simulação não confiável devido aos grandes gradientes de pressão gerados [7]. Assim, a velocidade média do escoamento foi utilizada como velocidade de entrada no runner.

A velocidade de inicial de $20 \mathrm{~m} / \mathrm{s}$ foi então escolhida pois representa a velocidade média aproximada no runner quando o motor opera em 8000 RPM. Porém, conforme enunciado acima, o motor pode cehgar a 13000 RPM, e o escoamento real no runner é um escoamento pulsante, assim, as velocidades presentes neste conduto são maiores que a velocidade média considerada. Assim, velocidades maiores como 40, 60 e $80 \mathrm{~m} / \mathrm{s}$ foram também ensaiadas a fim de visualizar a dependência do descolamento em função da velocidade.

A intensidade de turbulência na entrada do runner foi simulada como 5\%, um valor considerável para entrada de um conduto, mas que condiz com a natureza do escoamento real [6]. 


\section{Resultados}

Para a solução destas equações, utilizou-se a plataforma da ANSYS CFX para as três etapas da simulação: geração da malha, simulação, e pós tratamento, Vale a pena ressaltar que a malha simulada foi uma malha 3D de aproximadamente 1.4 milhões de elementos.

A principal análise feita foi em cima dos campos de velocidade e de pressão, assim como os vetores de velocidade no fim da curvatura do runner. Uma redução na velocidade na região interna da curva, apresentando ou não recirculação era a situação esperada de ser encontrada. Em conjunto com essa redução na velocidade, uma variação da pressão na parede da região interna do conduto indicaria uma possível recirculação, assim como uma variação abrupta na pressão na região da parede indicaria um descolamento do fluido.

Tomaram-se duas linhas de referência: a primeira linha é a linha de menor raio de curvatura do runner na sua seção meridional. A figura 6 mostra a evolução de pressão ao longo desta linha. Conforme esperado, há uma diminuição da pressão na região da curvatura e esta ao passo que a velocidade média do escoamento aumenta. Como a pressão de referência é de 1 atm, os valores negativos simbolizam reduções em relação a esta pressão de referência.

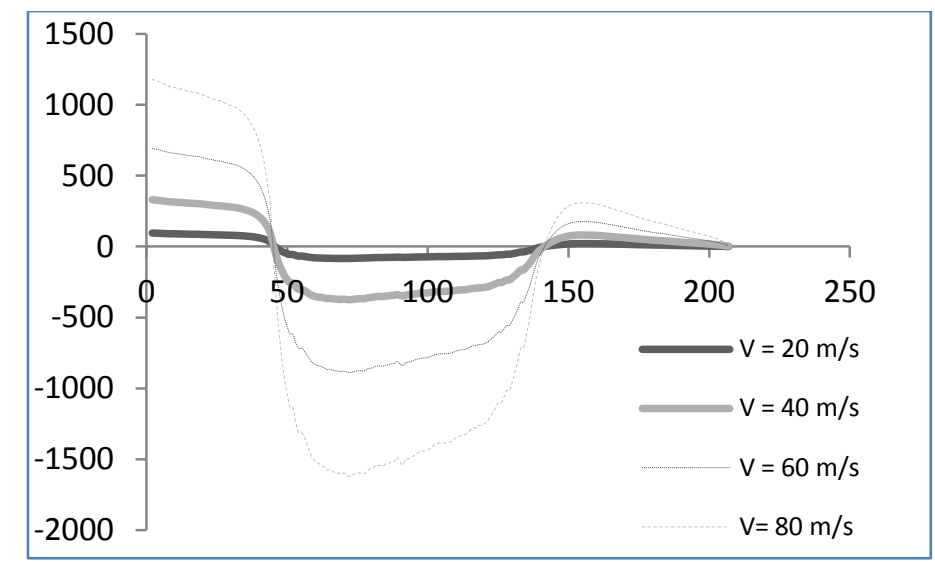

Figura 6. Pressão ao longo dos pontos de menor raio de curvatura do runner (região interna do raio de curvatura)

De maneira análoga, a figura 7 retrata a distribuição de pressão ao longo da linha externa do raio de curvatura (região de maior raio de curvatura). Como era de se esperar, ela apresenta valores positivos de pressão em relação à pressão de referência. Essa gradiente de pressão entre os pontos das figuras 6 e 7 gera uma resultante no fluido, que garante que o mesmo mude de direção.

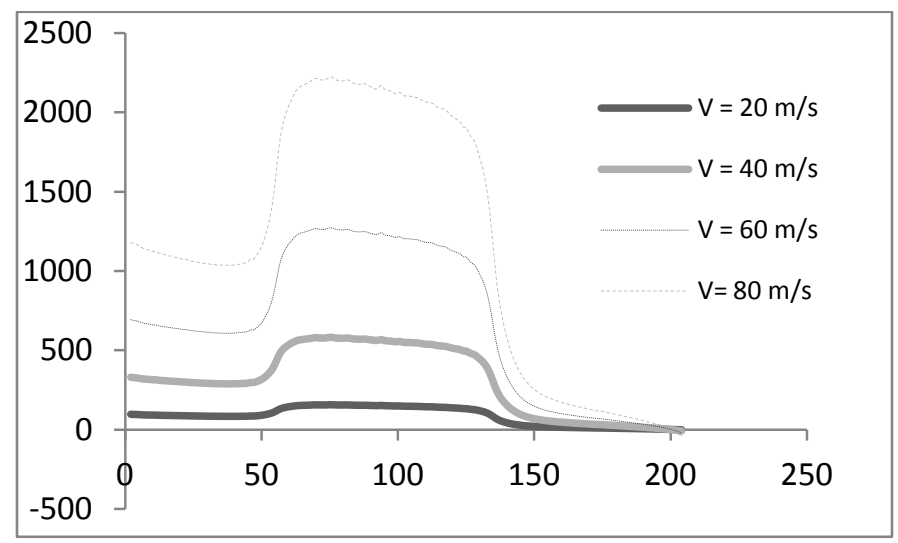


Outro ponto interessante de análise é comparar o campo de velocidade na seção final do runner, fim de sua curvatura e começo do trecho reto, que pode ser vista em detalhe na figura 8 (nota-se que o runner desta figura possui trechos retos maiores que o projetado para o veículo de Fórmula SAE, tanto na montante quanto na jusante do escoamento; isto foi feito para garantir uma simulação mais estável e confiável na região de curvatura - região de interesse).

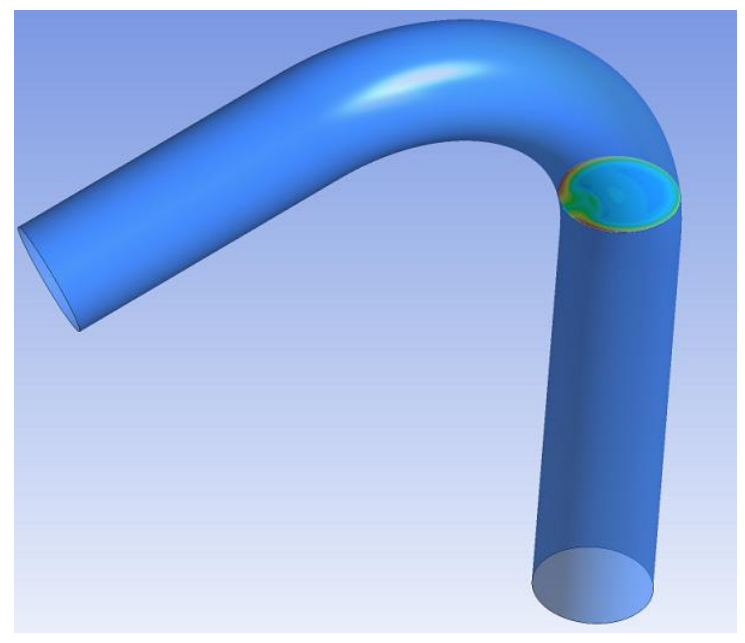

Figura 8. Runner simulado, com suas extremidades alongadas para garantir uma simulação mais estável na região de curvatura, enfatizando a região de análise dos vetores de velocidade

As figuras 9, 10, 11 e 12 mostram o campo de velocidade na região descrita na figura 8 (fim do raio de curvatura).

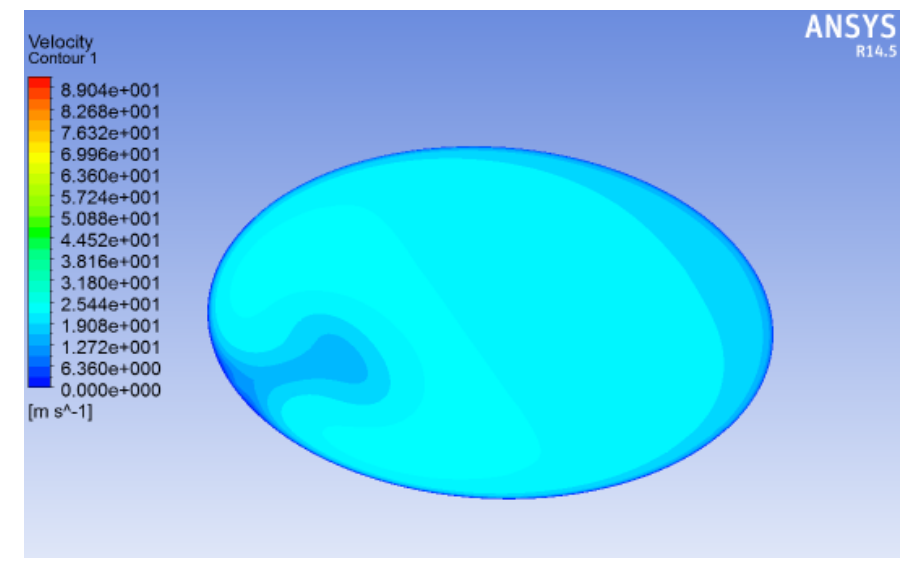

Figura 9. Campo de velocidade simulado com velocidade média do escoamento de $20 \mathrm{~m} / \mathrm{s}$ 


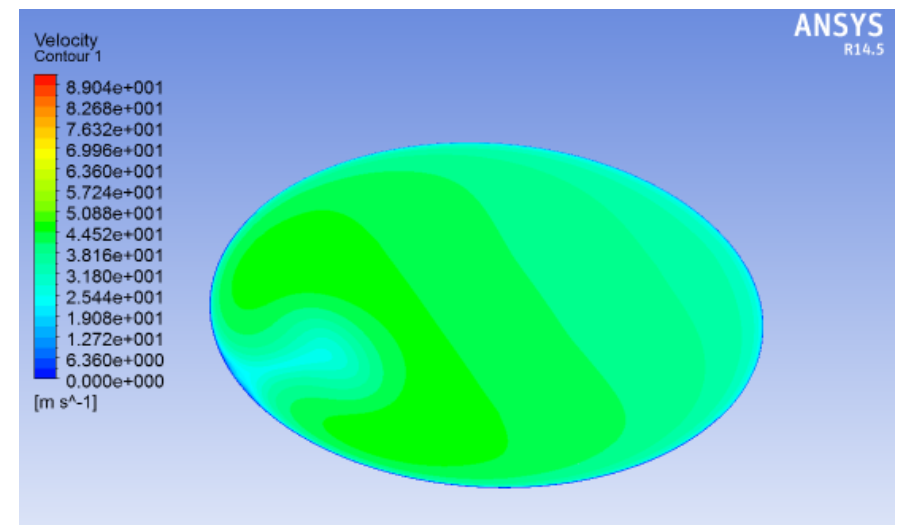

Figura 10. Campo de velocidade simulado com velocidade média do escoamento de $40 \mathrm{~m} / \mathrm{s}$

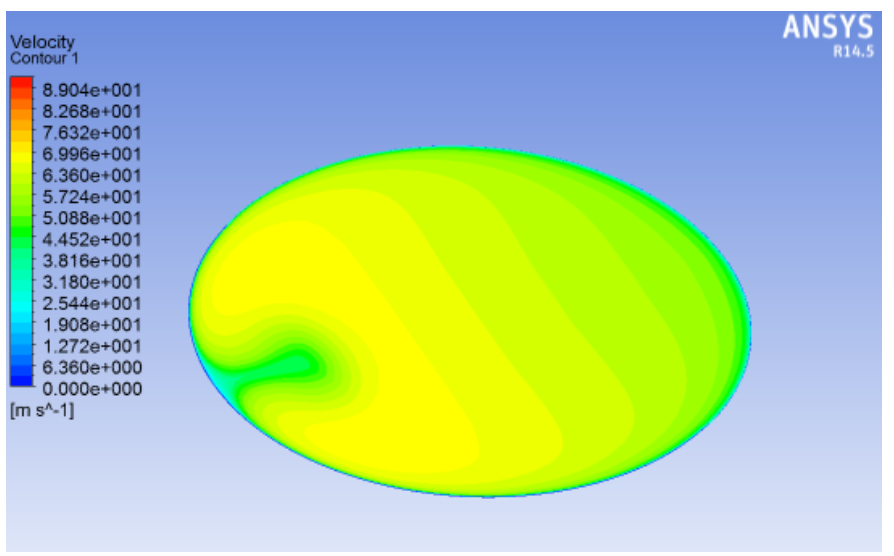

Figura 11. Campo de velocidade simulado com velocidade média do escoamento de $60 \mathrm{~m} / \mathrm{s}$

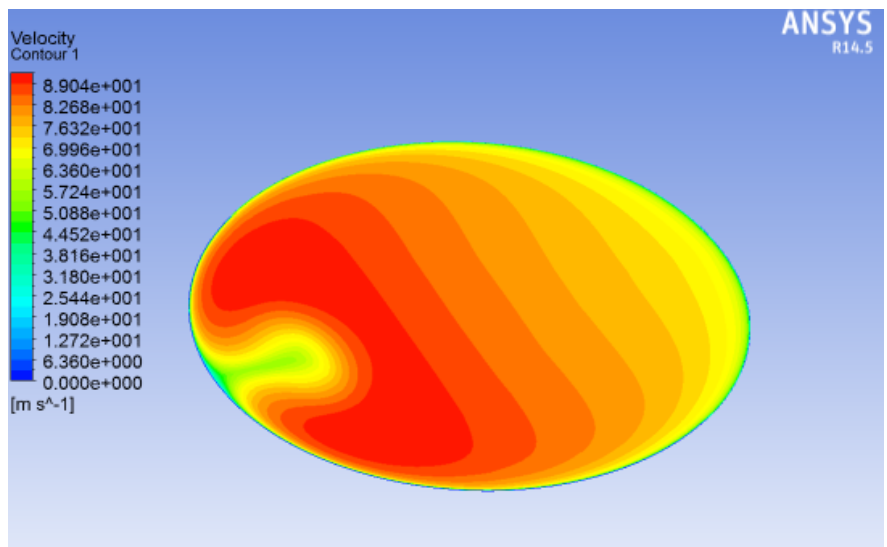

Figura 12. Campo de velocidade simulado com velocidade média do escoamento de $80 \mathrm{~m} / \mathrm{s}$

Por fim, foram analisados os vetores de velocidades para os mesmos planos (e mesmas vistas) das figuras 9 a 12. 


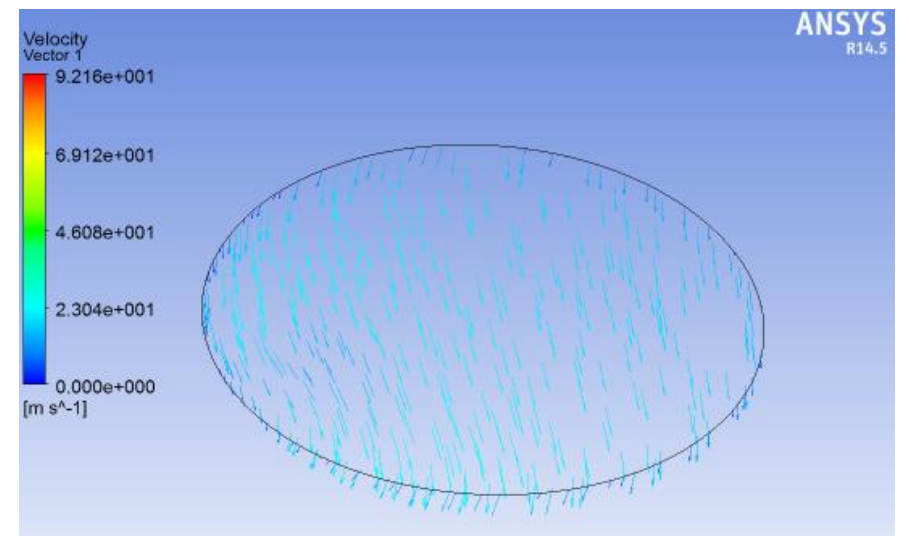

Figura 13. Vetores de velocidade simulados com velocidade média do escoamento de $20 \mathrm{~m} / \mathrm{s}$

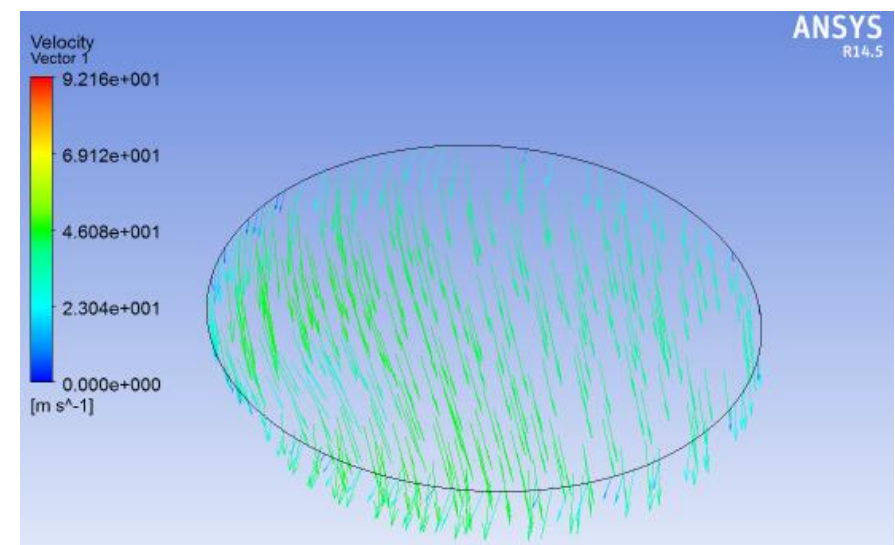

Figura 14. Vetores de velocidade simulados com velocidade média do escoamento de $40 \mathrm{~m} / \mathrm{s}$

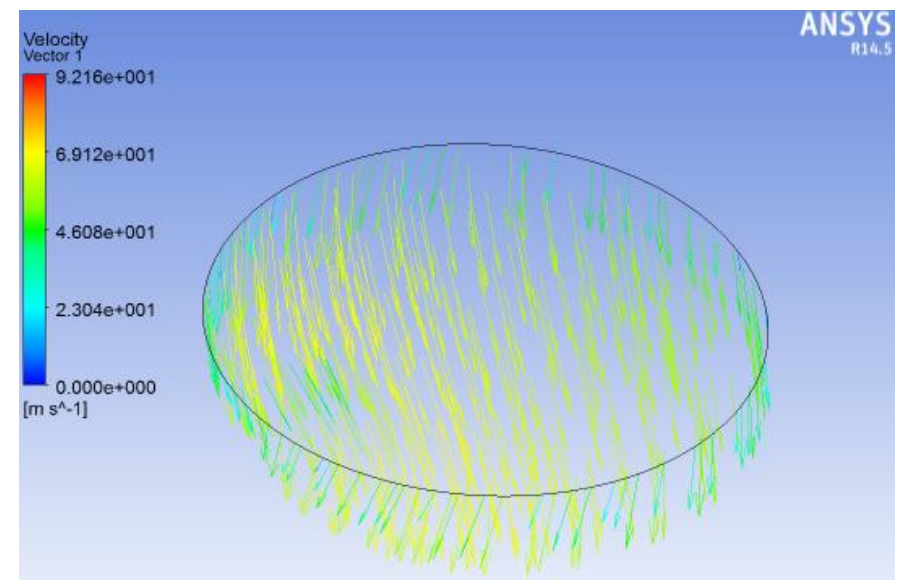

Figura 15. Vetores de velocidade simulados com velocidade média do escoamento de $60 \mathrm{~m} / \mathrm{s}$ 


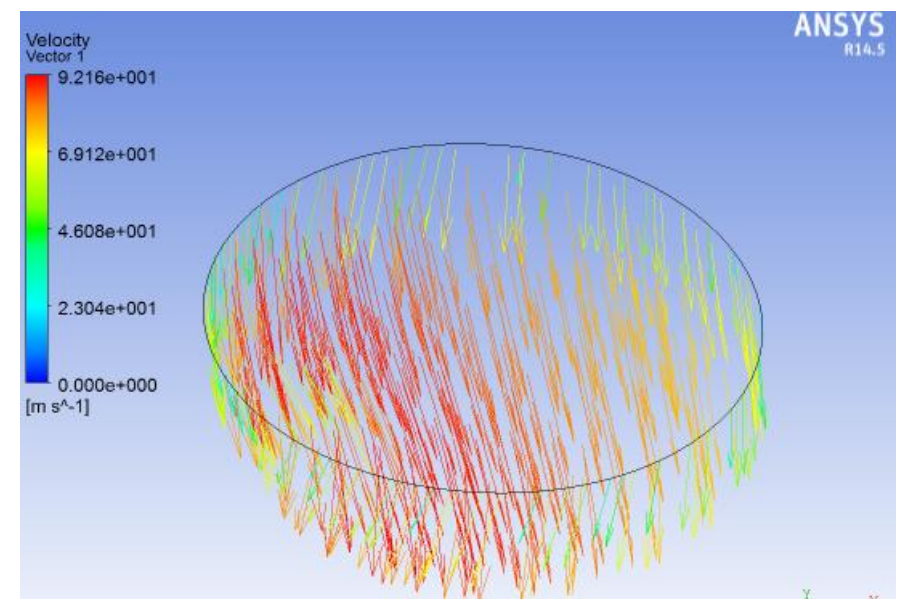

Figura 16. Vetores de velocidade simulados com velocidade média do escoamento de $80 \mathrm{~m} / \mathrm{s}$

\section{Conclusões e comentários}

A análise dos valores de pressão ao longo das duas linhas meridionais, internas e externas, do raio de curvatura do runner (figuras 6 e 7) não mostrou nenhum ponto de descolamento do fluido, mas forneceu as grandezas de variação de pressão a qual o fluido está sujeito e enfatizou a variação de pressão em função do aumento da velocidade média do escoamento. Quanto maior a velocidade maior a variação de pressão, o que é intuitivo. $\mathrm{O}$ que não é intuitivo é a não linearidade deste aumento (aumentos de velocidade causam aumentos cada vez maiores na diferença de pressão).

Ainda que não tenha sido notada nenhuma recirculação, muito menos um descolamento do fluido em relação à parede na região interna do raio de curvatura, pode-se perceber pela análise do campo de velocidade um alto rotacional, indicado pelo gradiente de velocidade na seção do fim da curvatura do runner (figuras 9 a 12). Tal rotacional se deve ao fato da velocidade na região interna do raio de curvatura ser consideravelmente menor que a velocidade média do escoamento. Conforme teorizado anteriormente, a mudança de direção do escoamento causou essa diminuição de velocidade, gerando uma situação semelhante à retratada na figura 5. Assim, o fluido teve sua área útil para escoamento reduzida, o que explica valores de velocidade a cima da média na região central da seção transversal (para garantir a conservação da massa uma vez que a simulação foi feita com fluxo de ar constante).

Possivelmente com um aumento da velocidade média do escoamento, ou também uma diminuição do raio de curvatura do runner, a diminuição da velocidade na região interna do raio de curvatura seria mais pronunciada, chegando ao ponto de causar uma recirculação e possivelmente um descolamento do ar, o que acarretaria uma diminuição da seção transversal útil para o escoamento do ar ainda maior.

A diminuição da seção transversal útil para o escoamento é responsável pelo aumento da perda de carga localizada, o que em termos práticos, representa para o motor do veículo, uma perda de potência, afinal, o fluido encontra um estrangulamento na jusante do runner, limitando assim a quantidade de ar que pode ser admitida pelo motor (deve-se ter em mente que a condição de contorno para simulação foi de fluxo constante, mas no motor em operação, o que ocorre é que existe uma diferença de pressão entre a montante e a jusante do runner, assim, ao passo que na simulação o estrangulamento gerou um aumento da velocidade na região do estrangulamento, no casso real, o estrangulamento causaria uma redução no fluxo de ar). 
Deve-se ressaltar que o motor, operando em condições normais, possui gradientes de pressão da ordem de 500 Bar/s [2], na região próxima à válvula, e a velocidade do escoamento só pode ser considerada constante na região à montante do plenum. Assim, a análise feita com velocidade constante do escoamento não representa em sua totalidade o real escoamento, deixando de representar os efeitos dos gradientes de pressão. Entende-se porém, que os gradientes de pressão apenas deixariam o escoamento ainda mais instável e colaborariam, em conjunto com um maior fluxo instantâneo, para um possível descolamento.

Assim, a primeira suspeita, que motivou este trabalho, pode ser até o momento explicada: as oscilações de torque e rotação que o motor do veículo de fórmula SAE da equipe Poli Racing, da Escola Politécnica da Universidade de São Paulo, eram causadas por um estrangulamento do ar na jusante do runner.

Para solucionar tal problema, a primeira abordagem seria tentar explorar ao máximo as dimensões disponíveis para o sistema de admissão, tentando diminuir assim, a curvatura do runner mas mantendo o comprimento do mesmo. Uma segunda abordagem seria o projeto de um novo runner com dispositivos passivos que evitassem tal descolamento, a saber: aletas direcionadoras ou divisórias internas longitudinais, soluções encontradas em sistemas de refrigeração [8,9]. Uma terceira opção seria o refazer o projeto da entrada de ar, em conjunto com a exaustão, de forma a modificar o comprimento do runner (isso geraria uma mudança na frequência de ressonância ótima, e por isso o projeto do sistema de exaustão deveria ser igualmente refeito a fim de garantir a mesma eficiência volumétrica do motor) e consequentemente, modificando o seu raio de curvatura.

\section{Referencias}

\section{[1] Regra Fórmula SAE 2011}

[2] Banco de dados, Equipe Poli Racing, da Escola Politécnica de São Paulo

[3] Heywood, John B, “Fundamentals of Internal Combustion Engine”, Mcraw- Hill, 1988, New York

[4] Munson B. R, “Fundamentos da Mecânica dos fluidos”, 4ed. Edgard Blücher

[5] Chambers, T. L . and Wilcox, D . C. (1977), "Critical Examination of Two-Equation Turbulence Closure Models for Boundary Layers," AIAA Journal, Vol. 15, No. 6

[6] Reynolds, W. C. (1987), "Fundamentals of Turbulence for Turbulence Modeling and Simulation," In Lecture Notes for von Karman Institute ,AGARD Lecture Series No. 86, pp. 1-66, New York

[7] Bradshaw, P. (1972), "The Understanding and Prediction of Turbulent Flow," The Aeronautical Journal, Vol. 76

[8] SMACNA (Sheet Metal and Air Conditioning Contractors' National Association) - HVAC Systems, Duct Design, edição de 2006

[9] ASHRAE (American society of Heating, Refrigerating, Air-Conditioning Engineers), Handbook of Fundamentals, ed. 2009 
Page 17 of 17 